\title{
BMJ Open Pioglitazone use and Parkinson's disease: a retrospective cohort study in Taiwan
}

\author{
Hsiu-Feng Wu, ${ }^{1}$ Li-Ting Kao, ${ }^{1,2}$ Jui-Hu Shih, ${ }^{2,3}$ Hui-Han Kao, ${ }^{1}$ Yu-Ching Chou, ${ }^{4}$ \\ I-Hsun Li, ${ }^{2,3}$ Senyeong Kao ${ }^{1,4,5}$
}

To cite: Wu H-F, Kao L-T, Shih J-H, et al. Pioglitazone use and Parkinson's disease: a retrospective cohort study in Taiwan. BMJ Open 2018;8:e023302. doi:10.1136/ bmjopen-2018-023302

- Prepublication history and additional material for this paper are available online. To view these files, please visit the journal online (http://dx.doi. org/10.1136/bmjopen-2018023302).

I-HL and SK contributed equally.

Received 30 March 2018

Revised 25 June 2018

Accepted 27 July 2018

Check for updates

(C) Author(s) (or their employer(s)) 2018. Re-use permitted under CC BY-NC. No commercial re-use. See rights and permissions. Published by BMJ.

${ }^{1}$ Graduate Institute of Life Sciences, National Defense Medical Center, Taipei, Taiwan

${ }^{2}$ Department of Pharmacy

Practice, Tri-Service General Hospital, Taipei, Taiwan

${ }^{3}$ School of Pharmacy, National

Defense Medical Center, Taipei,

Taiwan

${ }^{4}$ School of Public Health,

National Defense Medical

Center, Taipei, Taiwan

${ }^{5}$ Graduate Institute of Medical

Science, National Defense

Medical Center, Taipei, Taiwan

Correspondence to

Prof. Senyeong Kao;

kao@ndmctsgh.edu.tw

\section{ABSTRACT}

Objectives Many researchers have expected pioglitazone to serve as an effective neuroprotective agent against Parkinson's disease (PD). Therefore, we conducted this cohort study to investigate the association between pioglitazone use and PD by using a large Asian populationbased dataset in Taiwan.

Design Retrospective cohort study.

Setting Taiwan.

Participants 7906 patients with diabetes who had received pioglitazone were defined as the study cohort, and 7906 matched patients with diabetes who had not received pioglitazone were defined as the comparison cohort.

Primary and secondary outcome measures We tracked each patient individually over a 5-year follow-up period to identify those diagnosed as having PD during this period. We performed Cox proportional hazard regression analyses to evaluate the HRs for PD between the study and comparison cohorts.

Results The findings indicated that among the sampled patients, PD occurred in 257 (1.63\%): 119 (1.51\%) pioglitazone users and $138(1.75 \%)$ non-users. The adjusted HR for PD within the follow-up period was 0.90 (95\% Cl: 0.68 to 1.18) in the patients who had received pioglitazone compared with the matched patients who had not received pioglitazone. Moreover, this study revealed that pioglitazone use was not associated with PD incidence in men (HR: $1.06,95 \% \mathrm{Cl}: 0.71$ to 1.59$)$ or women (HR: $0.84,95 \% \mathrm{Cl}: 0.61$ to 1.15$)$.

Conclusions This study did not find the relationship between pioglitazone use and PD incidence, regardless of sex, among an Asian population of patients with diabetes.

\section{INTRODUCTION}

Parkinson's disease (PD) is a prevalent progressive neurodegenerative disorder estimated to affect approximately 7.5 million individuals worldwide. ${ }^{1-3}$ Patients with this disease commonly experience such symptoms as muscular rigidity, bradykinesia, tremor and postural instability. ${ }^{4-6}$ Many studies have indicated that these motor complications and other relevant symptoms may contribute to severe disabilities and substantially influence patients' quality of life. ${ }^{78}$ However, to date,

\section{Strengths and limitations of this study}

This study was a retrospective cohort study using a large Asian population-based dataset in Taiwan.

- The homogeneity of our study sample may have protected against confounding by ethnicity or race.

- The dataset used in this study provided sufficient sample size and statistical power.

- Selection bias that commonly occurs in observational studies was eliminated in this study.

- Some lifestyle data were unavailable in the dataset.

no clear effective or safe prevention strategy against PD has been demonstrated. Levodopa and dopamine agonists are recognised as available treatments for PD and could improve patients' clinical symptoms; nevertheless, long-term use of these treatments is limited by adverse effects and may not reverse the development of PD. ${ }^{9-11}$

Diabetes mellitus is a common metabolic disease which is characterised by the chronic hyperglycaemia. ${ }^{12}$ To date, many prior studies have considered that diabetes and PD may share similar pathophysiology. ${ }^{13}{ }^{14}$ Some recent epidemiological studies also observed the connection between diabetes and PD. ${ }^{15-17}$ Pioglitazone-a thiazolidinedione derivative-is a frequently administered treatment for diabetes mellitus ${ }^{18}$ and is considered to have high affinity for peroxisome proliferator-activated receptor- $\gamma($ PPAR- $\gamma) .{ }^{19}$ Many clinical studies have demonstrated that this therapeutic agent consistently reduces patients' serum levels of glucose and free fatty acid. ${ }^{20}$ Recently, increasing in vitro and in vivo evidences indicate that PPAR- $\gamma$ agonists may have neuroprotective effects. ${ }^{21-24}$ Therefore, many investigators have expected that pioglitazone could selectively stimulate PPAR- $\gamma$, inhibit the production of proinflammatory cytokines and serve as an effective neuroprotective agent against PD. ${ }^{25} 26$ 
To date, only a few studies conducted in Western countries have investigated whether pioglitazone can reduce the risk of PD. ${ }^{27-30}$ Moreover, although PD is a multifactorial disease that might be affected by genetic factors, ethnicity and environmental factors, no study has assessed the therapeutic benefits of pioglitazone among patients with PD in an Asian population. Furthermore, relevant results in previous studies have been inconsistent. Therefore, the present cohort study was conducted to explore the association between pioglitazone use and PD by using data from a nationwide population-based database in Taiwan.

\section{METHODS \\ Database}

The sample in this population-based cohort study was constructed using administrative claims data from the Taiwan Longitudinal Health Insurance Database 2005 (LHID2005). The LHID2005 contains the data of 1 million individuals randomly selected from all enrollees of Taiwan's National Health Insurance (NHI) programme in 2005 ( $\mathrm{n}=25.68$ million); specifically, the database contains the medical records and registry files of the selected enrollees. The NHI programme which offers comprehensive and affordable medical services for the Taiwanese population was founded in 1995, and approximately $99.9 \%$ of all Taiwanese citizens are registered in the programme. The LHID2005 provides de-identified secondary data to researchers in Taiwan for academic purposes.

\section{Study sample}

This retrospective cohort study involved a study cohort and comparison cohort. For the study cohort, we first selected 8381 patients diagnosed as having diabetes (International Classification of Diseases, Ninth Revision, Clinical Modification (ICD-9-CM): 250) between January 2002 and December 2008 who had received pioglitazone; we defined the first date of receipt of prescribed pioglitazone as the index date in this cohort. We then excluded patients aged under 40 years $(n=382)$ because the prevalence of PD is extremely low in this age group, and we further excluded 93 patients with a medical history of $\mathrm{PD}$ prior to the index date. The final sample in the study cohort consisted of 7906 patients with diabetes who had received prescriptions of pioglitazone.

For the comparison cohort, we selected 7906 other patients with diabetes from the LHID2005 who did not receive prescriptions of pioglitazone (one comparison patient per patient who received pioglitazone). Additionally, we constructed the comparison cohort by matching patients with diabetes who did not receive pioglitazone with patients in the study cohort based on sex, age group (40-49, 50-59, 60-69, 70-79, and $\geq 80$ years) and year of the index date, and we identified the first date of diabetes diagnosis in the matched year as the index date of each comparison patient. The comparison patients had not received pioglitazone since the founding of the NHI programme in 1995, and none had received a diagnosis of PD prior to the index date.

\section{Outcome measures}

This population-based cohort study investigated the association between pioglitazone use and subsequent PD status in patients with diabetes. Patients with PD were identified based on ICD-9-CM code 332. To define patients with diabetes subsequently diagnosed with $\mathrm{PD}$, each patient was individually tracked for a 5-year follow-up period following the index date. This study further conducted a dose-dependent analysis to investigate whether pioglitazone exposure strength was associated with PD. The cumulative defined daily dose (cDDD) was calculated as the sum of dispensed DDD of pioglitazone during 5-year study period in this study. We categorised the patients receiving pioglitazone into two levels according to the median dose (median cDDD=365).

\section{Statistical analysis}

We conducted all analyses in this study by using SAS for Windows (V.9.4, SAS Institute, Cary, North Carolina, USA). $\mathrm{X}^{2}$ tests were performed to investigate differences in geographical region (northern, central, southern and eastern Taiwan), urbanisation level (five levels, with level 1 being the most and level 5 being the least urbanised), monthly income, hypertension, hyperlipidaemia, depressive disorder, insomnia, stroke and head injury between the study and comparison cohorts. We used stratified Cox proportional hazard regressions to evaluate the HR of PD during the follow-up period between the study and comparison cohorts. Statistical significance was defined as a two-sided $\mathrm{p}$ value of $<0.05$.

\section{Patient and public involvement}

Patients or public were not involved in the development of the research question, outcome measures, study design and recruitment/conducting of the present study.

\section{RESULTS}

This population-based study involved 7906 patients with diabetes who had received prescriptions of pioglitazone as the study cohort and 7906 matched patients who had not been prescribed pioglitazone as the comparison cohort. The mean age in the study cohort was $61.2 \pm 10.6$ years, and that in the comparison cohort was $61.1 \pm 10.9$ years $(p=0.484)$. The distribution of demographic characteristics in both cohorts is shown in table 1. After matching for sex, age group and index year, we observed significant differences between the study and comparison cohorts in terms of geographical region $(\mathrm{p}=0.001)$, urbanisation level $(\mathrm{p}<0.001)$, hypertension $(\mathrm{p}<0.001)$, hyperlipidaemia $(p<0.001)$, depressive disorder $(p=0.014)$, insomnia $(\mathrm{p}=0.008)$, aspirin use $(\mathrm{p}<0.001)$, statins use $(\mathrm{p}<0.001)$ and angiotensin receptor blockers use $(\mathrm{p}<0.001)$. In 
Table 1 Demographic characteristics and comorbidities of patients with diabetes mellitus, stratified by whether or not patients received pioglitazone $(n=15812)$

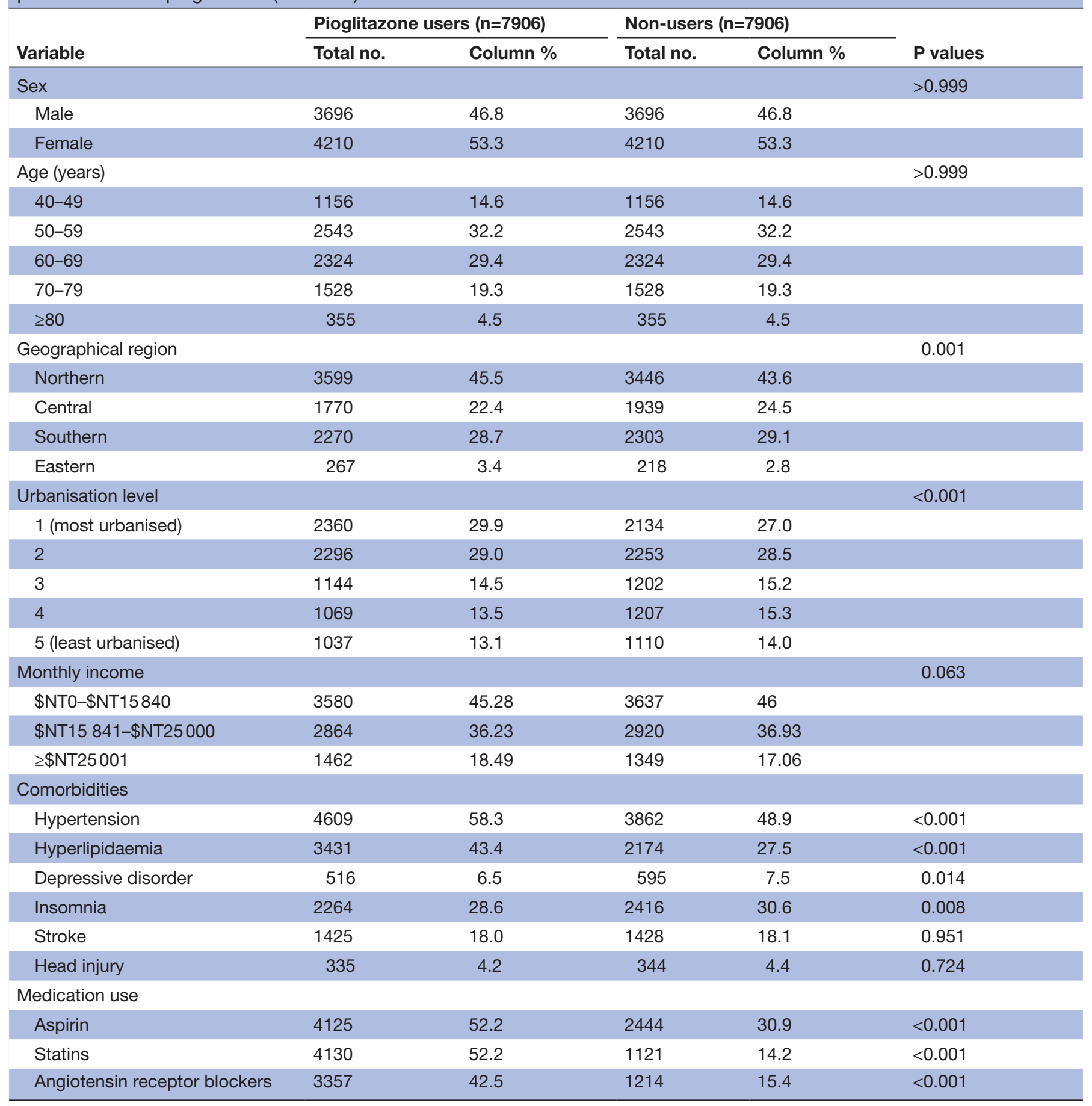

The average exchange rate in 2015 was US $\$ 1.00=\$ N T 30$.

NT, New Taiwan.

addition, we observed no significant differences in monthly income, stroke or head injury between the two cohorts.

Table 2 provides a comparison of PD incidence during the 5-year follow-up period between the study cohort and comparison cohort, revealing that among the sampled patients, PD occurred in 257 (1.63\%): 119 (1.51\%) pioglitazone users and $138(1.75 \%)$ non-users. Table 2 presents a comparison of the HRs for PD incidence in patients with diabetes who were pioglitazone users and those who were not pioglitazone users. The crude HR was 0.86 (95\% CI: 0.67 to 1.10 ) for the study patients compared with the comparison patients during the follow-up period. The adjusted HR for PD occurrence within 5 years following the index date was 0.90 (95\% CI: 0.68 to 1.18) in patients with diabetes who had received pioglitazone compared 
Table 2 HRs for Parkinson's disease among patients with diabetes mellitus during a 5-year follow-up period, stratified by whether or not patients received pioglitazone

\begin{tabular}{|c|c|c|c|}
\hline $\begin{array}{l}\text { Following incidence of } \\
\text { Parkinson's disease }\end{array}$ & Total sample $(n=15812)$ & Pioglitazone users $(n=7906)$ & Non-users $(n=7906)$ \\
\hline \multicolumn{4}{|l|}{ Five-year follow-up period } \\
\hline Crude HR $(95 \% \mathrm{Cl})$ & -- & 0.86 (0.67 to 1.10$)$ & 1.00 \\
\hline Adjusted HR* $(95 \% \mathrm{Cl})$ & -- & 0.90 (0.68 to 1.18$)$ & 1.00 \\
\hline
\end{tabular}

The adjusted HR was calculated by a Cox proportional hazard regression stratified by sex, age group and the index year. *Adjusted for geographical region, urbanisation level, monthly income, hypertension, hyperlipidaemia, depressive disorder, insomnia, stroke, head injury, aspirin use, statins use and angiotensin receptor blockers use.

with those who had not, after adjustment for geographical region, urbanisation level, monthly income, hypertension, hyperlipidaemia, depressive disorder, insomnia, stroke, head injury, aspirin use, statins use and angiotensin receptor blockers use. Furthermore, the findings regarding the covariate-adjusted HRs for Parkinson's disease among the sampled patients were displayed in online supplementary table 1 .

Table 3 presents the HRs for PD incidence between patients with diabetes who were pioglitazone users and patients who were non-users in terms of sex. The results revealed that pioglitazone use was not associated with PD incidence in both men and women with diabetes. The adjusted HRs for PD incidence were 1.08 (95\% CI: 0.72 to 1.61 ) and 0.84 (95\% CI: 0.61 to 1.16$)$, respectively, for men and women with diabetes who had received pioglitazone.

Table 4 shows the findings regarding dose-dependent analysis. It reveals the HRs for Parkinson's disease among patients with diabetes during a 5-year follow-up period, stratified by the cDDD of pioglitazone. We failed to observe the relationship between pioglitazone use and $\mathrm{PD}$ incidence in both high and low cumulative dose of pioglitazone. The adjusted HRs for PD occurrence were 0.94 (95\% CI: 0.66 to 1.34$)$ and 0.82 (95\% CI: 0.59 to $1.15)$, respectively, for patients receiving high cumulative dose of pioglitazone $(\geq 365 \mathrm{cDDD})$ and low cumulative dose of pioglitazone (<365 cDDD).

\section{DISCUSSION}

This nationwide retrospective cohort study failed to observe the association between pioglitazone use and PD after adjusting for geographical location, monthly income, urbanisation level, hypertension, hyperlipidaemia, depressive disorder, insomnia, stroke and head injury. Moreover, this study did not observe the association between pioglitazone use and PD among male and female patients. Because experimental studies have increasingly observed that pioglitazone could suppress proinflammatory cytokine production and may have neuroprotective effects, many researchers have expected that administering pioglitazone could be an effective therapeutic strategy for treating PD. ${ }^{21-26}$ However, only a few recent studies conducted in Western countries have explored the association between pioglitazone use and PD. ${ }^{27-30}$ Additionally, the findings of related studies have been inconsistent.

This population-based cohort study observed no association between pioglitazone use and PD risk among patients with diabetes mellitus, regardless of sex group. The results of our study are similar to those of other studies that have attempted to evaluate the efficacy of pioglitazone for treating PD. For instance, one multicentre, doubleblind, randomised trial (phase 2) conducted in the USA to analyse 210 patients revealed that pioglitazone use was unable to alter the progression of PD in the early stages. Another observational study conducted in the USA that analysed 29397 patients on Medicare revealed that the

Table 3 HRs for Parkinson's disease among sampled patients according to sex group

\begin{tabular}{|c|c|c|c|c|}
\hline \multirow[b]{2}{*}{$\begin{array}{l}\text { Following incidence of } \\
\text { Parkinson's disease }\end{array}$} & \multicolumn{2}{|l|}{ Male $(n=7392)$} & \multicolumn{2}{|l|}{ Female $(n=8420)$} \\
\hline & $\begin{array}{l}\text { Pioglitazone users } \\
(n=3696)\end{array}$ & Non-users $(n=3696)$ & $\begin{array}{l}\text { Pioglitazone users } \\
(n=4210)\end{array}$ & Non-users $(n=4210)$ \\
\hline Yes, n (\%) & $50(1.35)$ & 49 (1.33) & 69 (1.64) & $89(2.11)$ \\
\hline Crude HR (95\% Cl) & 1.02 (0.69 to 1.51$)$ & 1.00 & 0.77 (0.56 to 1.06$)$ & 1.00 \\
\hline
\end{tabular}

The adjusted HR was calculated by a Cox proportional hazard regression stratified by age group and the index year.

*Adjusted for geographical region, urbanisation level, monthly income, hypertension, hyperlipidaemia, depressive disorder, insomnia, stroke, head injury, aspirin use, statins use and angiotensin receptor blockers use. 
Table 4 HRs for Parkinson's disease among patients with diabetes mellitus during a 5-year follow-up period, stratified by pioglitazone cumulative dose

\begin{tabular}{|c|c|c|c|}
\hline \multirow{2}{*}{$\begin{array}{l}\text { Following incidence of } \\
\text { Parkinson's disease }\end{array}$} & \multicolumn{3}{|c|}{ Pioglitazone cumulative dose } \\
\hline & $\geq 365$ cDDD $(n=3957)$ & $<365$ cDDD $(n=3949)$ & 0 cDDD $(n=3957)$ \\
\hline \multicolumn{4}{|l|}{ Five-year follow-up period } \\
\hline Yes, n (\%) & $55(1.39)$ & $64(1.62)$ & $138(1.75)$ \\
\hline Crude HR $(95 \% \mathrm{Cl})$ & 0.79 (0.58 to 1.09$)$ & 0.93 (0.69 to 1.25$)$ & 1.00 \\
\hline Adjusted $\mathrm{HR}^{\star}(95 \% \mathrm{Cl})$ & 0.94 (0.66 to 1.34$)$ & $0.82(0.59$ to 1.15$)$ & 1.00 \\
\hline
\end{tabular}

*Adjusted for age, sex, geographical region, urbanisation level, monthly income, hypertension, hyperlipidaemia, depressive disorder, insomnia, stroke, head injury, aspirin use, statins use and angiotensin receptor blockers use. cDDD, cumulative defined daily dose.

HR for PD among thiazolidinedione users (including patients who used pioglitazone, troglitazone and rosiglitazone) was 1.09 (95\% CI: 0.71 to 1.66$)$ compared with sulfonylurea users. ${ }^{28}$ According to the relevant findings of our study and those of previous studies, pioglitazone use is not associated with PD incidence. In other words, pioglitazone might not reduce the risk of PD.

Relevant results obtained in two previous studies are inconsistent with our findings. ${ }^{29}{ }^{30}$ One of these studies was a retrospective cohort study that used data sourced from the Clinical Practice Research Datalink in the UK. ${ }^{29}$ That study revealed that thiazolidinedione users (including patients prescribed pioglitazone or rosiglitazone) were at a lower risk of PD than were matched users of other antidiabetic drugs. ${ }^{29}$ The other of the aforementioned two studies-conducted in Norway-observed that thiazolidinedione users were at a significantly lower risk of PD than were metformin only users (HR: $0.72 ; 95 \%$ CI: 0.55 to 0.94$).{ }^{30}$ These contradictory findings could be the result of several inherent limitations, such as ethnicity variance, information deficiency and study strategy difference. For instance, both of the aforementioned two studies analysed Western patient populations. ${ }^{29} 30$ By contrast, the present study analysed an Asian population to investigate the association between pioglitazone use and PD. In previous literature, the frequency of the PPAR- $\gamma$ Pro12Ala polymorphism varies from $2 \%$ to $25 \%$ depending on race/ethnicity. The frequency is relatively high in Caucasians (about 20\%); however, it is as low as $1 \%-3 \%$ in Asian and African population. ${ }^{31}$ Accordingly, genetic and environmental factors in Asian populations may be different from those in Western populations. In addition, the aforementioned study conducted in Norway did not consider some potential risk factors and comorbidities in the analyses ${ }^{30}$; such an information deficiency could affect the connection between pioglitazone use and PD. Furthermore, the aforementioned study conducted in the UK indicated that the observed preventive association was limited to cases of thiazolidinedione treatment and did not consider relative long-term benefits. ${ }^{29}$ Moreover, further analysis revealed that pioglitazone use did not reduce the risk of PD. ${ }^{29}$

The primary strength of our study is its population-based design. The large database used is representative of the entire Taiwanese population, and thus, selection biaswhich commonly occurs in observational studies-was eliminated. Furthermore, the characteristics of the LHID2005 provided sufficient statistical power to investigate the association between pioglitazone use and PD among patients with diabetes mellitus. Second, this study selected patients with diabetes mellitus as the comparison cohort; this design strategy may have prevented effects due to diabetes mellitus. Third, most patients involved in this cohort study were Han Chinese; the homogeneity of our study sample may have protected against confounding by ethnicity or race ${ }^{32}{ }^{33}$ Ethnicity may affect the association between pioglitazone use and PD incidence.

This population-based cohort study has several limitations. First, lifestyle data such as those related to tobacco smoking, body mass index, pesticide exposure, caffeine intake, alcohol consumption and physical activity are unavailable in the LHID2005. These factors were considered to be associated with PD development and may have affected the relevant findings of our study. ${ }^{34}$ Second, data of some patients with mild symptoms of PD may not be in the LHID2005, because such patients might consider relevant treatments non-essential and thus not directly seek Taiwan NHI medical services. Third, even though the diabetes control status may affect the association between pioglitazone use and PD, the laboratory data regarding glycosylated haemoglobin are unavailable in the LHID2005. Thus, we could not estimate the potential effects of the patients' diabetes control status in this study. Ultimately, our study investigated only the association between pioglitazone and PD incidence. The LHID2005 does not contain records of the Unified Parkinson's Disease Rating Scale (UPDRS). Consequently, we were unable to ensure whether pioglitazone use slows down the PD progression.

In summary, this retrospective cohort study failed to determine the relationship between pioglitazone use and PD incidence, regardless of sex, among an Asian population of patients with diabetes. Pioglitazone may not be an efficacious agent to prevent the incidence of PD. Nevertheless, additional large epidemiological studies considering the association between pioglitazone use and PD severity (eg, that determined based on the UPDRS) are 
required to determine whether pioglitazone use could decelerate PD progression or mitigate PD symptoms.

Contributors H-FW, SK, I-HL: conceived of this study and participated in study design. L-TK, J-HS: carried out the statistical analysis. Y-CC, H-HK: verified the analytical methods. H-FW, SK, I-HL, L-TK, J-HS, Y-CC, H-HK: helped to draft the manuscript. All authors reviewed the manuscript.

Funding The authors have not declared a specific grant for this research from any funding agency in the public, commercial or not-for-profit sectors.

Competing interests None declared.

Patient consent Not required.

Provenance and peer review Not commissioned; externally peer reviewed.

Data sharing statement № additional data available.

Open access This is an open access article distributed in accordance with the Creative Commons Attribution Non Commercial (CC BY-NC 4.0) license, which permits others to distribute, remix, adapt, build upon this work non-commercially, and license their derivative works on different terms, provided the original work is properly cited, appropriate credit is given, any changes made indicated, and the use is non-commercial. See: http://creativecommons.org/licenses/by-nc/4.0/.

\section{REFERENCES}

1. Ross GW, Abbott RD. Living and dying with Parkinson's disease. Mov Disord 2014;29:1571-3.

2. Pringsheim T, Jette N, Frolkis A, et al. The prevalence of Parkinson's disease: a systematic review and meta-analysis. Mov Disord 2014;29:1583-90.

3. Beitz JM. Parkinson's disease: a review. Front Biosci 2014;6:65-74.

4. Kalia LV, Lang AE. Parkinson's disease. Lancet 2015;386:896-912.

5. Bosboom JL, Wolters ECh. Psychotic symptoms in Parkinson's disease: pathophysiology and management. Expert Opin Drug Saf 2004;3:209-20.

6. Lew M. Overview of Parkinson's disease. Pharmacotherapy 2007;27:155S-60.

7. Hechtner MC, Vogt T, Zöllner Y, et al. Quality of life in Parkinson's disease patients with motor fluctuations and dyskinesias in five European countries. Parkinsonism Relat Disord 2014;20:969-74.

8. Okuma Y. Freezing of gait and falls in Parkinson's disease. $J$ Parkinsons Dis 2014;4:255-60.

9. Gazewood JD, Richards DR, Clebak K. Parkinson disease: an update. Am Fam Physician 2013;87:267-73.

10. Martin WR, Wieler M. Treatment of Parkinson's disease. Can J Neurol Sci 2003;30(Suppl 1):S27-33.

11. Olanow CW, Schapira AH. Therapeutic prospects for Parkinson disease. Ann Neurol 2013;74:337-47.

12. American Diabetes Association. Diagnosis and classification of diabetes mellitus. Diabetes Care 2010;33(Suppl 1):S62-9.

13. Lima MM, Targa AD, Noseda AC, et al. Does Parkinson's disease and type-2 diabetes mellitus present common pathophysiological mechanisms and treatments? CNS Neurol Disord Drug Targets 2014:13:418-28.

14. Santiago JA, Potashkin JA. System-based approaches to decode the molecular links in Parkinson's disease and diabetes. Neurobiol Dis 2014;72(Pt A):84-91.

15. De Pablo-Fernandez E, Goldacre R, Pakpoor J, et al. Association between diabetes and subsequent Parkinson disease: a recordlinkage cohort study. Neurology 2018;91.

16. Hu G, Jousilahti P, Bidel S, et al. Type 2 diabetes and the risk of Parkinson's disease. Diabetes Care 2007;30:842-7.

17. Schernhammer E, Hansen J, Rugbjerg K, et al. Diabetes and the risk of developing Parkinson's disease in Denmark. Diabetes Care 2011;34:1102-8.

18. Diamant M, Heine RJ. Thiazolidinediones in type 2 diabetes mellitus: current clinical evidence. Drugs 2003;63:1373-405.

19. Sakamoto J, Kimura H, Moriyama S, et al. Activation of human peroxisome proliferator-activated receptor (PPAR) subtypes by pioglitazone. Biochem Biophys Res Commun 2000;278:704-11.

20. Yki-Järvinen H. Thiazolidinediones. N Engl J Med 2004;351:1106-18.

21. Ridder DA, Schwaninger M. In search of the neuroprotective mechanism of thiazolidinediones in Parkinson's disease. Exp Neurol 2012;238:133-7.

22. Lecca D, Nevin DK, Mulas G, et al. Neuroprotective and antiinflammatory properties of a novel non-thiazolidinedione PPAR $\gamma$ agonist in vitro and in MPTP-treated mice. Neuroscience 2015;302:23-35.

23. Luna-Medina R, Cortes-Canteli M, Alonso M, et al. Regulation of inflammatory response in neural cells in vitro by thiadiazolidinones derivatives through peroxisome proliferator-activated receptor gamma activation. J Biol Chem 2005;280:21453-62.

24. Martin HL, Mounsey RB, Mustafa S, et al. Pharmacological manipulation of peroxisome proliferator-activated receptor $\gamma$ (PPAR $\gamma)$ reveals a role for anti-oxidant protection in a model of Parkinson's disease. Exp Neurol 2012;235:528-38.

25. Kalia LV, Kalia SK, Lang AE. Disease-modifying strategies for Parkinson's disease. Mov Disord 2015;30:1442-50.

26. Carta AR. PPAR- $\gamma$. therapeutic prospects in Parkinson's disease. Curr Drug Targets 2013;14:743-51.

27. NINDS Exploratory Trials in Parkinson Disease (NET-PD) FS-ZONE Investigators. Pioglitazone in early Parkinson's disease: a phase 2, multicentre, double-blind, randomised trial. Lancet Neurol 2015;14:795-803.

28. Connolly JG, Bykov K, Gagne JJ. Thiazolidinediones and parkinson disease: a cohort study. Am J Epidemiol 2015;182:936-44.

29. Brauer R, Bhaskaran K, Chaturvedi N, et al. Glitazone treatment and incidence of Parkinson's Disease among people with diabetes: a retrospective cohort study. PLoS Med 2015;12:e1001854.

30. Brakedal B, Flønes I, Reiter SF, et al. Glitazone use associated with reduced risk of Parkinson's disease. Mov Disord 2017;32:1594-9.

31. Meirhaeghe A, Amouyel P. Impact of genetic variation of PPARgamma in humans. Mol Genet Metab 2004;83:93-102.

32. de Lau LM, Breteler MM. Epidemiology of Parkinson's disease. Lancet Neurol 2006;5:525-35.

33. Van Den Eeden SK, Tanner CM, Bernstein AL, et al. Incidence of Parkinson's disease: variation by age, gender, and race/ethnicity. Am J Epidemiol 2003;157:1015-22.

34. Ascherio A, Schwarzschild MA. The epidemiology of Parkinson's disease: risk factors and prevention. Lancet Neurol 2016;15:1257-72. 